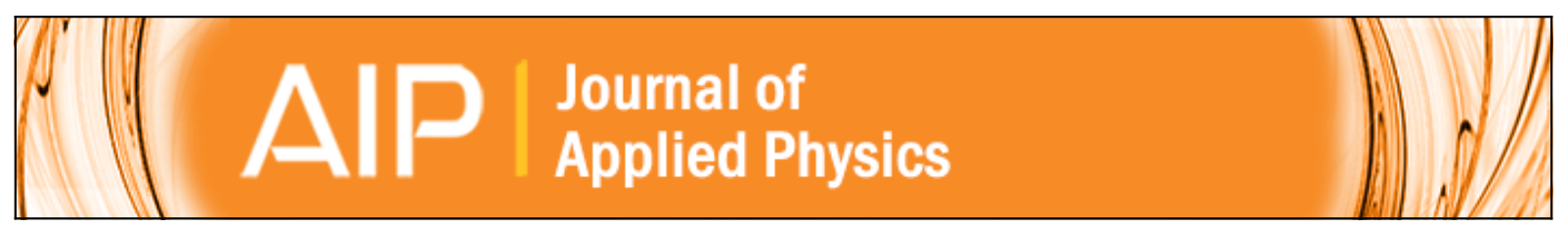

\title{
Arrays of membrane isolated yttrium-barium-copper-oxide kinetic inductance bolometers
}

M. A. Lindeman, J. A. Bonetti, B. Bumble, P. K. Day, B. H. Eom, W. A. Holmes, and A. W. Kleinsasser

Citation: Journal of Applied Physics 115, 234509 (2014); doi: 10.1063/1.4884437

View online: http://dx.doi.org/10.1063/1.4884437

View Table of Contents: http://scitation.aip.org/content/aip/journal/jap/115/23?ver=pdfcov

Published by the AIP Publishing

\section{Articles you may be interested in}

Jump properties of the tip magnetic field of a notch in a melt-processes yttrium-barium-copper-oxide bulk J. Appl. Phys. 107, 036102 (2010); 10.1063/1.3291116

Fabrication of complementary metal-oxide-semiconductor compatible semiconducting yttrium barium copper oxide uncooled infrared microbolometer arrays

J. Vac. Sci. Technol. B 25, 1315 (2007); 10.1116/1.2756551

Fabrication of a self-absorbing, self-supported complementary metal-oxide-semiconductor compatible micromachined bolometer

J. Vac. Sci. Technol. A 22, 842 (2004); 10.1116/1.1722404

Room temperature YBaCuO microbolometers

J. Vac. Sci. Technol. A 18, 635 (2000); 10.1116/1.582254

Low noise high- T c superconducting bolometers on silicon nitride membranes for far-infrared detection

J. Appl. Phys. 82, 4719 (1997); 10.1063/1.366327

\section{A.P $\left.\right|_{\text {Applied Physics }} ^{\text {Journal of }}$}

Journal of Applied Physics is pleased to announce André Anders as its new Editor-in-Chief 


\title{
Arrays of membrane isolated yttrium-barium-copper-oxide kinetic inductance bolometers
}

\author{
M. A. Lindeman, ${ }^{1, a)}$ J. A. Bonetti, ${ }^{1}$ B. Bumble, ${ }^{1}$ P. K. Day, ${ }^{1}$ B. H. Eom, ${ }^{2}$ W. A. Holmes, ${ }^{1}$ \\ and A. W. Kleinsasser ${ }^{1}$ \\ ${ }^{1}$ Jet Propulsion Laboratory, California Institute of Technology, Pasadena, California 91109, USA \\ ${ }^{2}$ California Institute of Technology, Pasadena, California 91125, USA
}

(Received 7 February 2014; accepted 9 June 2014; published online 20 June 2014)

\begin{abstract}
We are developing of arrays of membrane isolated resonator-bolometers, each with a kinetic inductance device (KID) to measure the temperature of the membrane. The KIDs are fabricated out of the high temperature superconductor YBCO to allow operation at relatively high temperatures. The bolometers are designed to offer higher sensitivity than sensors operating at $300 \mathrm{~K}$, but they require less expensive and lighter weight cooling than even more sensitive conventional superconducting detectors operating at lower temperatures. The bolometer arrays are applicable as focal planes in infrared imaging spectrometers, such as for planetary science missions or earth observing satellites. We describe the devices and present measurements of their sensitivity.

[http://dx.doi.org/10.1063/1.4884437]
\end{abstract}

\section{INTRODUCTION}

Bolometric infrared detectors based on high temperature superconductors (HTS) were proposed ${ }^{1}$ after the discovery of YBCO and developed into working devices. ${ }^{2,3}$ Development stopped, however, in part due to unavailability of low noise high $T_{c}$ multiplexed SQUID readouts for the HTS bolometers and the availability of competing uncooled microbolometers ${ }^{4}$ with much lower, but still useful sensitivity. However, new space borne instruments with higher spectral resolution, which image colder solar system objects, such as Europa or primitive bodies, would benefit from the 1-2 orders of magnitude higher sensitivity feasible with detectors made using HTS.

In the last decade, a new detector concept, the microwave kinetic inductance detector (MKID) was invented ${ }^{5}$ and has rapidly developed into large arrays that have been fielded on telescopes. ${ }^{6}$ The readout requires standard microwave components, rather than SQUIDs. Here, we describe prototype arrays of HTS bolometric detectors, shown in Fig. 1, that take advantage of this new readout method. The detector consists of a YBCO inductor, suspended on a membrane absorber, and embedded with an interdigitated capacitor in an LC circuit, coupled to a microwave feedline and operated in the temperature range up to $60 \mathrm{~K}$. Each absorber is connected to the heat sink, at temperature $T_{\mathrm{b}}$, by narrow legs which form a weak thermal leak with thermal conductance $G$. Ideally, the noise equivalent power (NEP) of the bolometer is determined by phonon noise ${ }^{7}$ of the weak link, which is $N E P_{\mathrm{PN}}=\sqrt{4 k_{\mathrm{B}} T_{\mathrm{b}}^{2} G}$. Absorption of power causes the temperature of the suspended membrane to increase, which changes the kinetic inductance of the YBCO and shifts the resonance frequency. A unique resonant frequency for each pixel is defined by varying the geometry of the interdigitated capacitors (IDCs), with resonances spaced over a $\mathrm{GHz}$ bandwidth. All pixels are read out simultaneously in the frequency domain as is done for other KID detectors.

\footnotetext{
${ }^{a)}$ Electronic mail: mark.a.lindeman@jpl.nasa.gov.
}

The devices are fabricated out of YBCO in order to operate at temperatures near $50 \mathrm{~K}$ while remaining well below the transition temperature of the superconductor, $90 \mathrm{~K}$. Measurements of thin film YBCO resonators ${ }^{8-10}$ show that resonators with quality factors in the thousands are plausible, even at temperatures well above $50 \mathrm{~K}$, making it possible to array hundreds of resonators on a single feedline. YBCO bolometers are expected to offer much higher sensitivity than room temperature detectors. For example, $500 \mu \mathrm{m}$ square YBCO bolometers with a thermal conductance $G$ of $25 \mathrm{nW} / \mathrm{K}$ at $50 \mathrm{~K}$ would result in a specific detectivity $D^{*}$ of $8 \times 10^{11}$ $\mathrm{cm} \mathrm{Hz} / 1 / 2 / \mathrm{W}$, which is much higher than in room temperature radiometers such as microbolometers ${ }^{11}$ or thermopiles, ${ }^{12}$ which have $D^{*}$ less than $1 \times 10^{9} \mathrm{~cm} \mathrm{~Hz}^{1 / 2} / \mathrm{W}$. YBCO bolometers do not require expensive, massive cooling systems as are required by conventional superconducting detectors that operate at a few kelvin or subkelvin temperatures. Inexpensive lightweight coolers ${ }^{13}$ are able to cool the bolometers down to $30 \mathrm{~K}$, and a passive system of baffles has been deployed in space ${ }^{14}$ that cools to less than $50 \mathrm{~K}$.

Arrays of these bolometers, coupled to imaging and wavelength dispersive optics such as an Offner grating or Fourier transform spectrometer, could be used to image the surface of cold planetary bodies while resolving hundreds

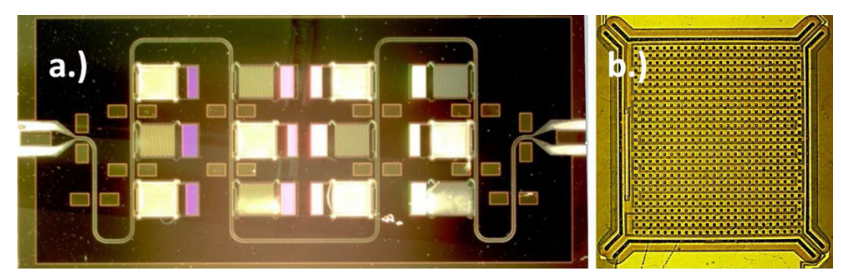

FIG. 1. YBCO KID bolometers. (a) Array "B" of 12 YBCO KID bolometers on a single feedline. Half of the bolometers are on isolated membranes (light colored squares), with the remaining half unsuspended. A KID lies on each membrane, and interdigitated capacitors, which appear as violet rectangles, lie next to the membranes. (b) A close up view of a YBCO KID, which meanders between the perforations in the membrane. The SiN legs in the corners define the thermal coupling $G$. (A test heater is also placed on the left edge of the membrane.) 
of different infrared wavelengths in the mid to far infrared (5 to $100 \mu \mathrm{m}$ ) with good spatial resolution (e.g., $225 \mathrm{~m}$ spatial resolution of the moon Europa from an platform $150 \mathrm{~km}$ above the surface). These data could be used to characterize the chemical, geological, and thermal properties of such bodies to provide information about origins and evolution of the solar system. Other applications include earth observing satellites, such as for measuring far infrared radiative cooling of the atmosphere to provide information relevant to climate models.

The temperature sensitivity of a KID bolometer depends on the how strong its impedance and resonance frequency vary respect to temperature $T$, and on the dissipation within the KID, which affects the internal quality factor $Q_{\mathrm{i}}$ of a resonator.

We parameterize the temperature sensitivity, using $\alpha_{\theta}$, which is the imaginary part of $\alpha$ defined in AC bolometer theory ${ }^{15,16}$

$$
\alpha_{\theta}=-2 Q_{i} \frac{T}{f_{r}} \frac{\partial f_{r}}{\partial T} .
$$

The sensitivity of the bolometers also depends on the coupling quality factor $Q_{\mathrm{c}}$, which is associated with dissipation outside of the bolometer. The quality factor $Q$ of a resonator is determined by both the internal and coupling quality factors $1 / Q=1 / Q_{\mathrm{i}}+1 / Q_{\mathrm{c}}$.

The YBCO bolometer's design minimizes sources of noise that typically affect KIDs. The noise from two level systems (TLSs), which is significant in subkelvin KIDs, decreases with temperature and so is not expected to be a problem for YBCO devices, which operate at much higher temperatures. Thermal quasiparticle noise is a form of internal thermal fluctuation noise (ITFN) associated with the exchange of energy between phonons and quasiparticles. It is suppressed by designing the thermal link $G$ such that the time heat takes to leak off the membrane is much longer than the thermal quasiparticle recombination time. In this design, the components on the membrane thermalize, acting like a single body with a single temperature, which suppresses ITFN as in other types of bolometers. ${ }^{17}$ Or in an equivalent description, recombination of quasiparticles on the membrane generates phonons that are not lost as in standard KIDs, but instead break up more quasiparticles, thereby increasing the effective quasiparticle lifetime and reducing quasiparticle noise.

Dissipation of power $P_{0}$ in the bolometer is associated with Johnson noise ${ }^{18}$ having noise equivalent power in the phase direction $^{15,16,19}$ of $N E P_{J} \sim \sqrt{\left(G T_{b} / P_{0} \alpha_{\theta}^{2}\right)} \cdot N E P_{P N}$. The dissipation noise may exceed Johnson noise due to creation quasiparticles with energy $\Delta$ by the microwave bias in the KID, which has been estimated ${ }^{20}$ to create shot noise with $N E P_{g 1}=\sqrt{4 \Delta P_{0}}$. In another model, ${ }^{19}$ quasiparticle generation energy comes directly from the microwave bias, resulting in additional voltage fluctuations with $N E P_{g 2} \sim \sqrt{\Delta / k_{\mathrm{B}} T \cdot N E P_{J}}$.

We typically apply a small bias power $P_{0}$, typically much less than $G T_{\mathrm{b}} / 50$, and $\Delta$ is less typically than $\sim 10 k_{\mathrm{B}} T_{\mathrm{b}}$. In this case, $N E P_{P N}$ exceeds the other aforementioned noise sources if

$$
\alpha_{\theta}>\sim 6 \sqrt{G T_{\mathrm{b}} / P_{0}} .
$$

(The above noise estimations are derived from AC bolometer theory ${ }^{19}$ assuming amplitude response, current dependence, and detuning are negligible and that $Q_{i} \cong Q_{c}$ ). Large values of $\alpha_{\theta}$ are required because the maximum bias power that can be applied to the bolometer is limited by the onset of the Duffing oscillator behavior, which occurs in over biased KIDs. ${ }^{20}$ Below, we present measurements of $\alpha_{\theta}$, and we describe how large $\alpha_{\theta}$ is required to be, given the observed limit on bias power $P_{0}$.

\section{FABRICATION}

Our bolometers are fabricated on $\mathrm{Si}$ substrates coated with a $250 \mathrm{~nm}$-thick layer of amorphous silicon nitride $\left(\mathrm{SiN}_{\mathrm{x}}\right)$, which is used for the KID membranes. Because YBCO does not grow on amorphous surfaces, ${ }^{21}$ we employ a textured, polycrystalline buffer layer consisting of $6-8 \mathrm{~nm}$ thick $\mathrm{Y}_{2} \mathrm{O}_{3}$ and 5-10 nm thick $\mathrm{MgO}$, both deposited by ion beam assisted deposition ${ }^{22-26}$ (IBAD), followed by $110 \mathrm{~nm}$ thick epitaxial $\mathrm{MgO}$ deposited at $500^{\circ} \mathrm{C}$. On top of the buffer layers is $50 \mathrm{~nm}$-thick YBCO with $50 \mathrm{~nm}$-thick Au, both deposited by evaporation in the same pump down. The YBCO and gold layers are patterned to define the KIDs, IDCs, wiring, and bond pads. Next, the silicon nitride is etched to define the shape of the membranes, with four narrow legs at the corners to attach the membrane to the substrate. At the same time, a grid of holes is etched into the membrane surrounding each $5 \mu \mathrm{m}$ wide KID meander. Finally $\mathrm{XeF}_{2}$ is used to etch away $\mathrm{Si}$ under the holes and laterally under the membranes. This leaves the KID suspended on the silicon nitride membrane, with the bolometers weak thermal link defined by the narrow legs as illustrated in Fig. 1(b). The membrane area is $500 \times 600 \mu \mathrm{m}^{2}$, with approximately $25 \%$ of the material absent due to the perforations in the membrane. The four legs each have an area of $15 \mu \mathrm{m}$ wide by $60 \mu \mathrm{m}$ long. We avoid etching the membranes of some of the bolometers to compare suspended bolometers to those lying on the silicon substrate. We describe results from inductively coupled arrays, as shown in Fig. 1(a). In these arrays, a trace in each resonator circuit lies adjacent to the feedline to provide inductive coupling. We measured square impedance of our YBCO films to be approximately $100 \Omega /$ sq just above the transition temperature. The meanders are roughly impedance matched to free space by designing the filling fraction of the meanders on the membrane to be about $50 \%$, doubling the effective square impedance for wavelengths longer than the pitch of the meanders.

\section{EXPERIMENT}

The YBCO arrays were tested in an IR Labs dewar, which was outfitted with microwave readout and cooled to $4.2 \mathrm{~K}$. The first tests were done on a bolometers from an array of resonators, labeled array "A." Measurements of the bolometer response to steps in the microwave bias, plotted in Fig. 2, showed that an unsuspended bolometer had a response decay time of $27 \mu$ s, while a suspended bolometer had a thermal decay time of $380 \mu$ s, due to weak thermal 


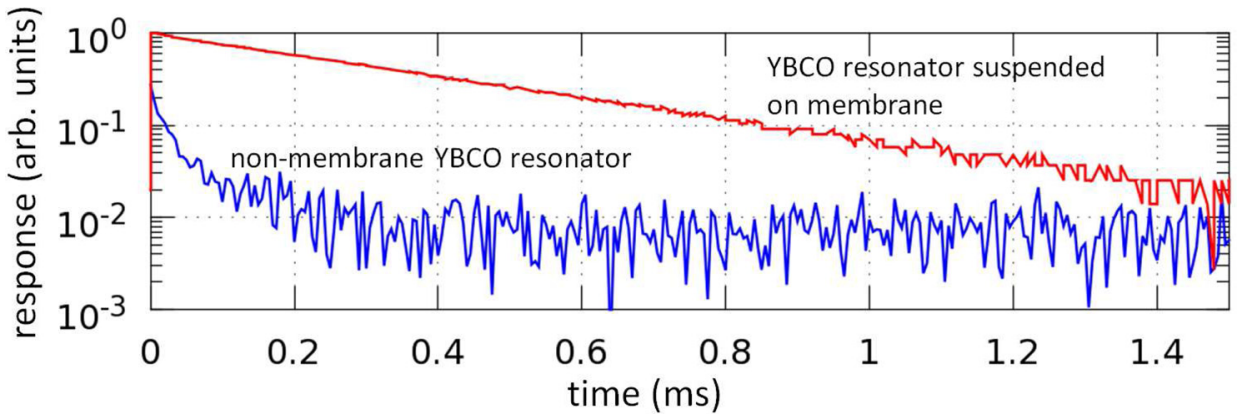

FIG. 2. Response time of a YBCO resonator bolometer compared to a similar unsuspended bolometer. The response time of the bolometer is increased due to the slow diffusion of heat out through the legs, resulting in a longer duration signal.

coupling through the legs. Using published values of thermal conductance and heat capacity of silicon nitride, ${ }^{27}$ the thermal time is estimated to be $\sim 500 \mu \mathrm{s}$.

The bolometer arrays were placed in an enclosed box on a stage with thermometry and a heater to heat it to higher temperatures for testing. A vector network analyzer was used to measure the transmission coefficient S21 of the resonators as a function of frequency, bath temperature, and bias power. The S21 data are normalized so that off resonance, transmission equals 1 and phase shifts from propagation delay on the cabling are removed. The resonances were fitted by a resonance mode ${ }^{28}$ to extract resonance frequency, and the quality factors, according to

$$
\begin{aligned}
S_{21} & =a\left(1-\frac{Q / \widehat{Q}_{\mathrm{e}}}{1+2 i Q \frac{f-f_{\mathrm{r}}}{f_{\mathrm{r}}}}\right), \text { where } \\
\operatorname{Re}\left[\frac{1}{\hat{Q}_{\mathrm{e}}}\right] & =\frac{1}{Q_{\mathrm{c}}}=\frac{1}{Q}-\frac{1}{Q_{\mathrm{i}}} .
\end{aligned}
$$

Measurements of a suspended YBCO KID from array "A" are plotted in Fig. 3. The results show sufficiently high $Q$ for temperatures in the $30 \mathrm{~K}$ to $40 \mathrm{~K}$ range, with $Q_{\mathrm{i}} \sim 1500$, $T=35 \mathrm{~K}, f_{\mathrm{r}}=930 \mathrm{kHz}$, and $\partial f_{\mathrm{r}} / \partial T=-3.5 \mathrm{kHz} / \mathrm{K}$. Using Eq. (1), we find $\alpha_{\theta}=400$. These test devices are under coupled to the feedline with $Q_{\mathrm{c}} \sim 32000$. This is compensated for by applying larger bias to the feedline.

Based on the results from array "A," we designed masks to pattern 2 in. wafers which are diced into 28 chips, each containing a bolometer array or other test device. In the new devices, the pattern of holes etched into the membranes was changed to include more, but smaller, perforations to facilitate higher yields of free standing membranes. The KID

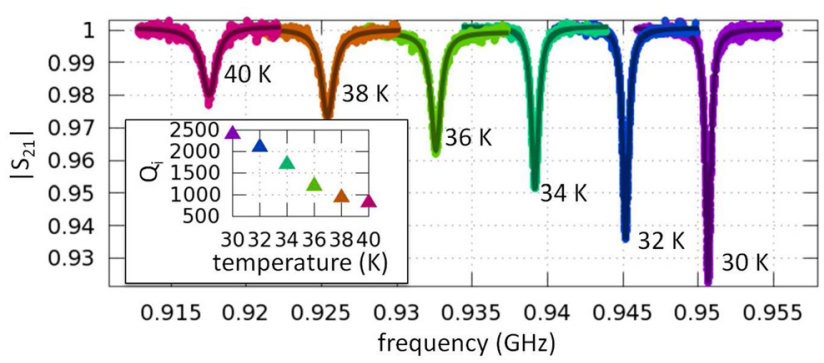

FIG. 3. Resonance vs. temperature of YBCO bolometer from array "A." The inset shows the corresponding measurements of the internal quality factor. The plot color identifies different bath temperatures. meanders were rerouted around the new hole pattern, but the dimensions of the meanders and width of the traces were kept the same. One of these arrays, array "B" of Fig. 1(a), was tested. The resulting measured values of $Q_{\mathrm{i}}$ are plotted in Fig. 4. Nine of the twelve resonances were detected, including functioning suspended and unsuspended bolometers. The coupling quality factors of these bolometers ranged from 23000 to 41000 , depending on the device.

The data in Fig. 4 indicate that most of array "B" of bolometers had internal quality typically between factors of 2200 to 3000 at $6 \mathrm{~K}$. Three of 12 resonators on the array were undetected, probably due to imperfect fabrication yield. The shifts in the resonance frequency relative to the resonance frequency at $5.4 \mathrm{~K}$ are plotted in Fig. 5 . At $17 \mathrm{~K}$, the frequencies are reduced by $0.5 \%$, corresponding to a kinetic inductance fraction of 0.01 . The resonance plotted in the inset plot of Fig. 4 with resonance frequency $f_{\mathrm{r}}=1.2 \mathrm{GHz}$,

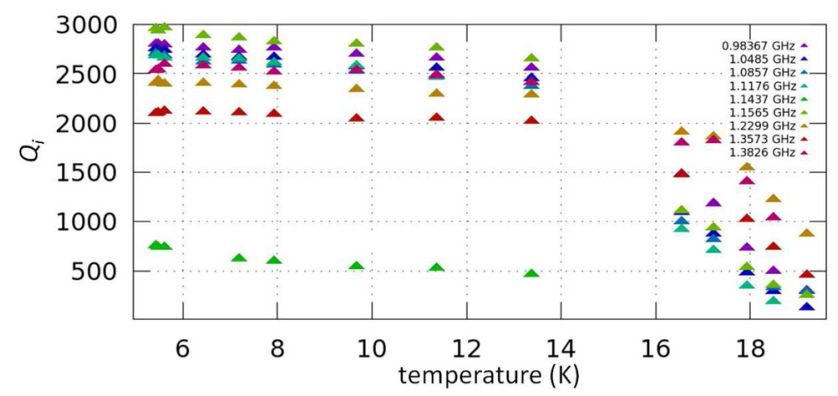

FIG. 4. The internal quality factors vs. temperature of YBCO KID bolometers from array "B." The colors correspond to different resonators with their resonance frequencies at $5.4 \mathrm{~K}$ listed on the right.

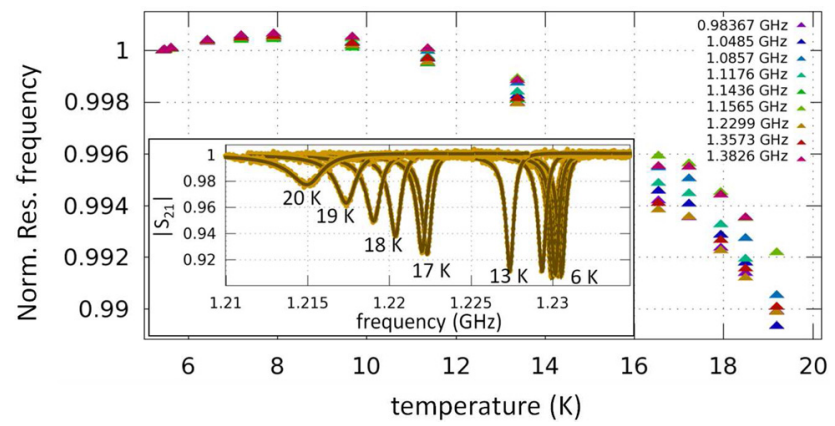

FIG. 5. Relative shifts in resonance frequency vs. temperature of YBCO KID bolometers from array "B." The resonance frequencies are normalized with respect to the resonance frequency at $5.4 \mathrm{~K}$. The colors correspond to different resonators with their resonance frequencies at $5.4 \mathrm{~K}$ listed on the right. The inset shows transmission measurements and best fits for the $1.2299 \mathrm{GHz}$ resonance. 


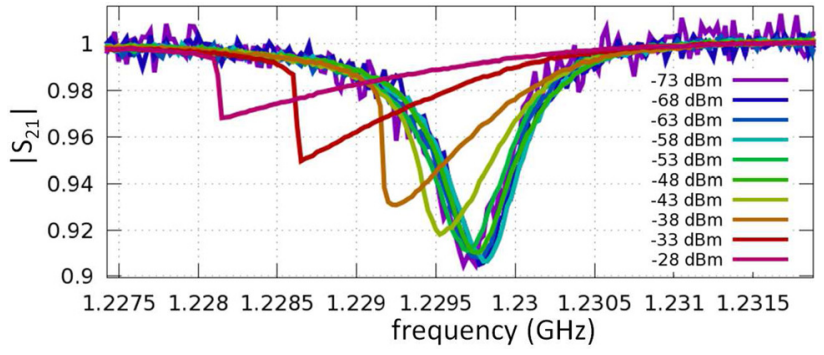

FIG. 6. Resonance shape vs. microwave bias power. The power applied to the feedline in each of the measurements is listed on the right. At resonance, the mismatch associated with $Q_{\mathrm{c}}=23000$ and $Q \mathrm{i}=2400$ results in an additional $8 \mathrm{~dB}$ of power attenuation into the bolometer. This device is relatively unaffected by the bias powers up to $-45 \mathrm{dBm}$ on the feedline or $-53 \mathrm{dBm}$ of power in the bolometer. At higher powers, the electric current significantly increases the kinetic inductance and the dissipation in the resonator.

had $Q_{\mathrm{i}}=1800$ and $\partial f_{\mathrm{r}} / \partial T=-2.3 \mathrm{kHz} / \mathrm{K}$ at $T=17 \mathrm{~K}$, resulting in $\alpha_{\theta}=120$. The other bolometers also had $\alpha_{\theta}$ near 100 at $17 \mathrm{~K}$. However, the quality factor of all the resonances started to decrease at roughly $16 \mathrm{~K}$, with the resonances becoming undetectable above $21 \mathrm{~K}$. We also observed that the transmission of the feedline decreased by $50 \%$ as we warmed the devices from $20 \mathrm{~K}$ to $50 \mathrm{~K}$. The decreased $\mathrm{Q}$ and feedline transmission also occurred in other arrays from the same wafer as array "B." These data suggest a problem with the surface impedance of the YBCO films, which occurred most significantly in Array "B" and other arrays from the same wafer. DC measurements of the feedline of array " $B$ " indicated it was superconducting up to a critical temperature of about $80 \mathrm{~K}$.

Measurements of resonator response vs. bias power in the KIDs of array "B" were conducted at $6 \mathrm{~K}$. The response of one of the resonators to bias power is plotted in Fig. 6 . These data indicated the resonances were not significantly distorted up to power $-53 \mathrm{dBm}$ (or $5 \mathrm{nW}$ ) in the bolometer. At higher power, the resonances became distorted by typical Duffing oscillator behavior. These measurements produced no distinguishable differences between the suspended and unsuspended resonators because the effects of Joule heating were small compared to Duffing oscillator distortion.

\section{DISCUSSION}

The internal quality factor of the YBCO devices is lower than standard MKIDs which may have Q values in the tens of thousands. However, the devices are sensitive due to the change in inductance with respect to temperature, resulting in large values of $\alpha_{\theta}$. Using Eq. (2), with the specified thermal conductance $25 \mathrm{nW} / \mathrm{K}$ at $50 \mathrm{~K}$ of the planetary science application described above, and bias power of $5 \mathrm{nW}$ from the experiment, we find, that $\alpha_{\theta}>100$ should provide sufficient gain to boost the phonon noise above other sources of noise. Because we measured much higher values of $\alpha_{\theta}$, we expect the YBCO bolometers will have sufficient sensitivity.

Measurements of the bolometer of array "A" demonstrated high sensitivity at temperatures between $30 \mathrm{~K}$ and $40 \mathrm{~K}$, which is in the range of inexpensive cryocoolers. YBCO microwave devices, such as microstrip resonators, ${ }^{8-10}$ have demonstrated low surface resistance and high quality factors at $77 \mathrm{~K}$ and frequencies of a few $\mathrm{GHz}$. We therefore expect the problem with feedline transmission and low $Q$ at higher temperatures, which is most significant in array "B," to be solvable with further investigation, allowing future devices to be operable at higher temperatures.

\section{ACKNOWLEDGMENTS}

This research was supported by an appointment to the NASA Postdoctoral Program at the Jet Propulsion Laboratory, administered by Oak Ridge Associated Universities through a contract with NASA. The research was carried out at the Jet Propulsion Laboratory, California Institute of Technology, under a contract with the National Aeronautics and Space Administration. IBAD deposition of $\mathrm{MgO}$ was performed by I-Beam Materials. The YBCO was deposited by Ceraco Ceramic Coating and patterned by Star Cryoelectronics. The authors would like to thank Vince Kotsubo for useful discussions.

${ }^{1}$ P. Richards, J. Clarke, R. Leoni, P. Lerch, S. Verghese, M. R. Beasley, T. H. Geballe, R. H. Hammond, P. Rosenthal, and S. R. Spielman, Appl. Phys. Lett. 54, 283 (1989).

${ }^{2}$ S. J. Berkowitz, A. S. Hirahara, K. Char, and E. N. Grossman, Appl. Phys. Lett. 69, 2125 (1996).

${ }^{3}$ A. Lee, J. Gildemeister, L. Shih-Fu, and P. Richards, IEEE Trans. Appl. Supercond. 7, 2378 (1997).

${ }^{4}$ W. R. Johnson, S. J. Hook, and S. M. Shoen, Opt. Lett. 37, 803 (2012).

${ }^{5}$ P. Day, H. LeDuc, B. Mazin, A. Vayonakis, and J. Zmuidzinas, Nature 425, 817 (2003).

${ }^{6}$ L. J. Swenson, P. K. Day, C. D. Dowell, B. H. Eom, M. I. Hollister, R. Jarnot, A. Kovács, H. G. Leduc, C. M. McKenney, R. Monroe, T. Mroczkowski, H. T. Nguyen, and J. Zmuidzinas, Proc. SPIE 8452, 84520P (2012).

${ }^{7}$ J. C. Mather, Appl. Opt. 21, 1125 (1982).

${ }^{8}$ W. Rauch, E. Gornik, G. Sölkner, A. A. Valenzuela, F. Fox, and H. Behner, J. Appl. Phys. 73, 1866 (1993).

${ }^{9}$ R. Schneider, A. G. Zaitsev, J. Geerk, G. Linker, F. Ratzel, and R. Smithey, Supercond. Sci. Technol. 15, 212 (2002).

${ }^{10}$ M. Skoryk, V. Pan, V. Tarasov, V. Flis, A. Filimonov, and S. Futimsky, in Phys. Eng. Microwaves, Millimeter, Submillim. Waves. MSMW 04 (Kharkov, 2004), pp. 418-420.

${ }^{11}$ W. Radford and R. Wyles, Proc. SPIE 3379, 22 (1998).

${ }^{12}$ A. Graf, M. Arndt, M. Sauer, and G. Gerlach, Meas. Sci. Technol. 18, R59 (2007).

${ }^{13}$ R. Unger and D. Keiter, AIP Conf. Proc. 710, 1404 (2004).

${ }^{14}$ J. A. Tauber, N. Mandolesi, and T. Banos, Astron. Astrophys. 520, A1 (2010).

${ }^{15}$ M. A. Lindeman, P. Khosropanah, and R. A. Hijmering, J. Appl. Phys. 113, 074502 (2013).

${ }^{16}$ M. A. Lindeman, B. H. Eom, P. K. Day, L. J. Swenson, R. Wernis, H. G. LeDuc, and J. Zmuidzinas, J. Low Temp. Phys. 176, 511 (2014).

${ }^{17}$ K. Kinnunen, Ph.D. thesis, University of Jyväskylä, 2011.

${ }^{18}$ H. Nyquist, Phys. Rev. 32, 110 (1928).

${ }^{19}$ M. A. Lindeman, "Resonator-bolometer theory, microwave read out, and kinetic inductance bolometers," J. Appl. Phys., submitted (2014).

${ }^{20}$ J. Zmuidzinas, Annu. Rev. Condens. Matter Phys. 3, 169 (2012).

${ }^{21}$ J. M. Phillips, J. Appl. Phys. 79, 1829 (1996).

${ }^{22}$ J. Harper and J. J. Cuomo, J. Vac. Sci. Technol. 21, 737 (1982).

${ }^{23}$ L. Yu, J. Harper, J. Cuomo, and D. Smith, Appl. Phys. Lett. 47, 932 (1985).

${ }^{24}$ R. Bradley, J. M. E. Harper, and D. A. Smith, J. Appl. Phys. 60, 4160 (1986).

${ }^{25}$ D. Fork, D. Fenner, A. Barrera, J. Phillips, T. Geballe, G. Connel, and J. B. Boyce, IEEE Trans. Appl. Supercond. 1, 67 (1991).

${ }^{26}$ C. Wang, K. Do, M. Beasley, T. Geballe, and R. Hammond, Appl. Phys. Lett. 71, 2955 (1997).

${ }^{27}$ B. L. Zink and F. Hellman, Solid State Commun. 129, 199 (2004).

${ }^{28}$ M. S. Khalil, M. J. A. Stoutimore, F. C. Wellstood, and K. D. Osborn, J. Appl. Phys. 111, 054510 (2012). 